\title{
Procedimentos de predição e efeitos da heterogeneidade de variâncias residuais dentro de tratamentos genéticos
}

\author{
Diego Tyszka Martinez ${ }^{1}$, Marcos Deon Vilela de Resende ${ }^{2}$, Antonio Rioyei Higa ${ }^{3}$, Reginaldo Brito da Costa ${ }^{1}$ \\ ${ }^{1}$ Universidade Federal de Mato Grosso (UFMT), Av. Fernando Corrêa da Costa, 2367, Boa Esperança, CEP 78.00-900, Cuiabá, MT, Brasil \\ ${ }^{2}$ Embrapa Florestas, Estrada da Ribeira, Km 111, CP 319, CEP 83.411-000, Colombo, PR, Brasil \\ ${ }^{3}$ Universidade Federal do Paraná (UFPR), Av. Lothário Meissner, 900, Jardim Botânico, CEP 80170-220, Curitiba, PR, Brasil
}

"Autor correspondente:

diegotyszka@hotmail.com

Termos para indexação:

Modelos lineares mistos

Parâmetros genéticos

Ganho com seleção

Index terms:

Mixed linear models

Genetic parameters

Selection gain

Histórico do artigo:

Recebido em 18 mar 2011

Aprovado em 17 ago 2011

Publicado em 30 set 2011

doi: 10.4336/2011.pfb.31.67.193
Resumo - O presente estudo objetivou comparar, via simulação, os procedimentos BLUP e BLUP-HET para predição dos valores genéticos sob heterogeneidade de variâncias residuais. Os dados aleatórios foram gerados em planilha eletrônica, considerando uma variância genética de 0,10 e variância residual variável, por número de clones, de forma que tivessem heterogeneidade de variâncias. Os valores genéticos reais e residuais foram somados à média 10 , obtendo assim os valores fenotípicos positivos. Utilizou-se o delineamento de blocos ao acaso, com 100 genótipos, uma planta por parcela, com 2, 5, 10 e 20 repetições. Os dados foram avaliados no software Selegen, obtendo os valores genéticos estimados pelos procedimentos BLUP e BLUP-HET e comparados com os valores genéticos reais. Nestas condições, o uso de duas e cinco repetições apresenta baixa precisão. Com herdabilidade próxima de $10 \%$, recomendase o uso de dez ou mais repetições para garantir maior precisão nas estimativas, não representando problema prático em casos de heterogeneidade de variâncias dentro de genótipos, podendo ser utilizado qualquer um dos procedimentos. Apesar disso, o procedimento BLUP-HET resulta em acurácias mais próximas do valor esperado, para a maioria dos casos avaliados, além de estimar o ganho com seleção mais próximo ao real.

\section{Prediction methods and heterogeneity effects of residual variances within genetic treatments in clone tests}

\begin{abstract}
The aim of this study was to compare, through simulation, the BLUP and BLUP-HET procedures for breeding values prediction under heterogeneity of residual variances. Random data were generated with spread sheet, considering a variance of 0,10 and variable residual variance, by number of clones, in order to provide heterogeneity of variances. Actual and residual breeding values obtained were added to the mean 10, in order to obtain positive phenotypic values. Experimental design was random blocks with 100 genotypes, one plant per plot and 2, 5, 10 and 20 repetitions. Data were evaluated using Selegen software, obtaining estimated breeding values through BLUP and BLUP-HET procedures which were compared to actual breeding values. In this study, using two and five repetitions showed low accuracy. With heritability close to $10 \%$, it is recommend to use ten or more repetitions in order to ensure greater accuracy in estimates. This represents no restrain in case of variances heterogeneity within genotypes, being both methods suitable. Nevertheless, for most of the cases, BLUP-HET results in accuracies closer to expected values. Furthermore, its estimates for selection gain are closer to real figures.
\end{abstract}




\section{Introdução}

Em experimentos de campo nos programas de melhoramento genético se deseja um alto grau de precisão experimental e, consequentemente, uma elevada acurácia (Resende \& Duarte, 2007). Esta precisão experimental e acurácia dependem da instalação adequada dos experimentos, da coleta de dados e da forma como estes dados são analisados. Segundo Resende (2002), quanto maior a acurácia, maior é a confiança na avaliação e na estimativa do valor genético. O processo de avaliação dos tratamentos genéticos em experimentos deve inferir sobre os valores genotípicos dos materiais e ordená-los com base nesses valores para uso no processo de seleção (Resende \& Duarte, 2007).

A escolha adequada do modelo estatístico para realizar a análise de um experimento e identificar os efeitos fixos e aleatórios para minimizar a interferência ambiental é fundamental para que sejam selecionados os melhores genótipos. Segundo Cruz et al. (2004), um efeito é considerado como fixo quando as conclusões a seu respeito valerem apenas para ele próprio e um efeito é considerado aleatório quando o material avaliado é uma amostra de uma população. A utilização dos efeitos fixos ou aleatórios irá definir os resultados obtidos na estimação dos componentes de médias e de variância e, consequentemente, interferir diretamente na acurácia de suas estimativas.

Segundo Marcelino \& Iemma (2000), a estimação dos componentes de variância utilizando modelos mistos e dados desbalanceados têm merecido atenção especial dos pesquisadores nas últimas décadas. Para estes autores, a maioria dos estudos tem considerado modelos com efeitos fixos, sendo que, em muitas situações, um ou mais fatores são de efeitos aleatórios, demonstrando a importância de modelos com efeitos aleatórios e modelos mistos. Ressaltam ainda que, quando a estimação dos componentes de variância é feita sobre dados desbalanceados, diferentes métodos podem ocasionar diferentes estimativas de um mesmo parâmetro.

Atualmente, o uso de modelos mistos é o mais indicado em programas de melhoramento genético. Neste caso, os blocos são tratados como de efeito fixo, por ser o principal estrato homogêneo para comparação de indivíduos, sendo que, neste caso, a comparação pode ser feita de maneira não viciada
(Resende, 2002). Para Resende (2007), a estimação dos componentes de variância pelo procedimento da máxima verossimilhança restrita (REML) e a predição dos valores genéticos pelo procedimento da melhor predição linear não viciada (BLUP) são considerados os procedimentos ótimos de estimação para dados balanceados ou desbalanceados.

Em modelos mistos frequentemente assume-se que a variância residual é a mesma para todas as observações. Porém, as diferenças na variância residual entre os indivíduos são comuns, tornando importante a inclusão dos efeitos dos resíduos heterogêneos nos modelos tradicionais (Rönnegard et al., 2010).

Em situações onde há heterogeneidade de variâncias, um procedimento BLUP que acomoda a heterogeneidade de variâncias deve ser priorizado (Resende \& Duarte, 2007). Segundo os autores, nesta situação, cada tratamento genético apresenta diferentes acurácias, coeficientes de determinação genéticos e variações residuais dentro do tratamento. Para este caso, o BLUPHET, que considera a heterogeneidade de variâncias residuais e estima os componentes de variância por genótipo deve ser priorizado.

A variabilidade residual existente no delineamento é de difícil análise, pois, quando se instala um experimento, alguns preceitos devem ser levados em consideração, dentre eles, homogeneidade dentro dos blocos. No caso de experimentos com plantas perenes, a necessidade de grandes áreas faz com que haja um maior risco de heterogeneidade ambiental, ocasionando erros nas estimativas.

O presente estudo objetivou comparar, via simulação, os procedimentos BLUP e BLUP-HET para predição dos valores genéticos sob heterogeneidade de variâncias residuais, visando identificar em que situações o uso do BLUP-HET é suficiente e necessário.

\section{Material e métodos}

Os dados para 100 clones não aparentados foram simulados, utilizando-se delineamento de blocos completos casualizados, uma planta por parcela, com 2 , 5,10 e 20 repetições e dez variáveis para cada número de repetições.

A geração dos números aleatórios foi realizada em planilha eletrônica, usando ferramenta de geração de números aleatórios. Utilizou-se a distribuição normal, com média zero e desvio padrão definido de acordo 
com o tipo de variância desejada. Neste procedimento, identifica-se o número de variáveis e a quantidade de números aleatórios desejados para esta variância e distribuição. Dessa forma, foram gerados números aleatórios com variâncias específicas, sendo 0,10 para as variâncias genéticas (supondo homogeneidade) e variâncias variáveis para os efeitos residuais, geradas por grupo de clones, de forma a obter variâncias heterogêneas. Neste caso, o efeito genético é repetido para o mesmo clone e o efeito do erro varia em todos os casos, não se repetindo. As variâncias utilizadas por respectivo grupo e número dos clones estão descritas na Tabela 1. Após serem gerados os dados, estes foram somados e adicionados à média 10 (evitando assim valores fenotípicos negativos), obtendo-se o valor fenotípico que foi utilizado nas análises e mantendo-se o valor genético real (VGR) para comparação.

Tabela 1. Distribuição das variâncias genéticas e residuais e herdabilidades associadas $\left(\mathrm{h}^{2}\right)$ à cada combinação de genótipos, para o respectivo grupo de clones.

\begin{tabular}{lccc}
\hline \multicolumn{1}{c}{ Clones } & $\begin{array}{c}\text { Variância } \\
\text { genética }\end{array}$ & $\begin{array}{c}\text { Variância } \\
\text { Residual }\end{array}$ & $\begin{array}{c}\mathbf{h}^{2} \text { resultante da } \\
\text { simulação }\end{array}$ \\
\hline Clones 1 a 5 & 0,10 & 0,95 & 0,10 \\
Clones 6 a 25 & 0,10 & 0,80 & 0,11 \\
Clones 26 a 75 & 0,10 & 0,70 & 0,13 \\
Clones 76 a 95 & 0,10 & 0,60 & 0,14 \\
Clones 96 a 100 & 0,10 & 0,50 & 0,17 \\
\hline
\end{tabular}

As predições dos valores genéticos pelas metodologias BLUP e BLUP-HET foram realizadas utilizando-se o Software Selegen-REML/BLUP (Resende, 2002). O modelo estatístico usado foi o de blocos ao acaso, teste de clones não aparentados, com uma planta por parcela, representado matematicamente pela expressão: $\mathrm{y}=\mathrm{Xr}$ $+\mathrm{Zg}+\mathrm{e}$; sendo y: vetor de dados; r: vetor dos efeitos de repetição (assumidos como fixos) somados à média geral; g: vetor dos efeitos genotípicos (assumidos como aleatórios); e: vetor de erros ou resíduos (aleatórios); X: matriz de incidência dos efeitos de repetição; Z: matriz de incidência dos efeitos genotípicos (Resende, 2007).

Os resultados dos componentes de variâncias (REML) foram obtidos através das seguintes fórmulas:

Herdabilidade individual no sentido amplo no bloco, ou seja, dos efeitos genotípicos:

$$
\widehat{h}_{g}^{2}=\frac{\widehat{\sigma}_{g}^{2}}{\widehat{\sigma}_{g}^{2}+\widehat{\sigma}_{e}^{2}}=\frac{\widehat{\sigma}_{g}^{2}}{\widehat{\sigma}_{f}^{2}}
$$

Sendo $\widehat{\sigma}_{g}^{2}$ - variância genotípica entre clones; $\hat{\sigma}_{e}^{2}$ - variância residual ou ambiental entre parcelas; $\hat{\sigma}_{f}^{2}$ variância fenotípica $\left(\widehat{\sigma}_{g}^{2}+\widehat{\sigma}_{e}^{2}\right)$.

Herdabilidade da média de clones, assumindo estande completo:

$$
\hat{h}_{m c}^{2}=\frac{\hat{\sigma}_{g}^{2}}{\hat{\sigma}_{g}^{2}+\frac{\hat{\sigma}_{e}^{2}}{b}}
$$

Sendo: $\widehat{\sigma}_{g}^{2}$ : variância genotípica entre clones; $\widehat{\sigma}_{e}^{2}$ : variância residual ou ambiental entre parcelas; $b$ : número de blocos.

Herdabilidade da média para o clone i (i variando de 1 a 100):

$$
\widehat{h}_{m c i}^{2}=\frac{\hat{\sigma}_{g}^{2}}{\hat{\sigma}_{g}^{2}+\frac{\hat{\sigma}_{e i}^{2}}{b}}
$$

Sendo: $\widehat{\sigma}_{g}^{2}$ : variância genotípica entre clones; $\widehat{\sigma}_{e i}^{2}$ - variância residual ou ambiental entre parcelas para o clone $\mathrm{i} ; b$ : número de blocos.

Acurácia da seleção de clones, assumindo estande completo:

$$
\text { Acclon }=\sqrt{h_{m c}^{2}}
$$

Sendo: $\widehat{h}_{m c}^{2}$ : herdabilidade da média de clones, assumindo estande completo.

Diferencial de seleção: $d s=m s-\mu$

Sendo: $m s$ : média dos clones selecionados; $\mu$ : média original do experimento.

O valor genotípico predito ( $v g$ ) de cada clone é dado pela herdabilidade da média de clones $\left(\hat{h}_{m c}^{2}\right)$ multiplicado pelo diferencial de seleção mais a média original. Quando não se adiciona a média original na fórmula, têm-se apenas os efeitos genotípicos $(g)$.

Para o procedimento BLUP, têm-se, no caso balanceado, assumindo homogeneidade de variâncias:

$g=\widehat{h}_{m c}^{2} \cdot(d s)$ : efeito genotípico.

$v g=\mu+g=\hat{h}_{m c}^{2} .(d s)+\mu$ : valor genotípico predito. 
Sendo: $\widehat{h}_{m c}^{2}$ : herdabilidade da média de clones, assumindo estande completo; $d s$ : diferencial de seleção; $g$ : efeito genotípico; $\mu$ : média original do experimento.

Para o procedimento BLUP-HET, têm-se, no caso balanceado:

$$
g=\widehat{h}_{m c i}^{2} \cdot(d s) \text { : efeito genotípico do clone i. }
$$

$v g=\mu+g=\widehat{h}_{m c i}^{2} \quad .(d s)+\mu$ : valor genotípico predito do clone $\mathrm{i}$.

Sendo: $\widehat{h}_{m c i}^{2}$ : herdabilidade da média para o clone i (i variando de 1 a 100); $d s$ : diferencial de seleção; $g$ : efeito genotípico; $\mu$ : média original do experimento.

As acurácias seletivas dos procedimentos BLUP e BLUP-HET foram obtidas utilizando a correlação de Pearson entre o valor genético real e os valores preditos pelos procedimentos BLUP e BLUP-HET. A acurácia esperada foi calculada através da seguinte fórmula (RESENDE, 2002):

$$
r_{\hat{g} g}=\left[\frac{b \widehat{h}_{g}^{2}}{1+(b-1) \hat{h}_{g}^{2}}\right] 1 / 2
$$

Sendo: $b$ : número de blocos; $\widehat{h}_{g}^{2}$ : herdabilidade individual no sentido amplo no bloco (dos efeitos genotípicos).

Comparou-se também o ganho com seleção pelos procedimentos BLUP e BLUP-HET, considerando-se os valores genéticos reais, com uma intensidade de seleção de $10 \%$ (dez clones). Primeiramente, ordenouse, pelo valor genético do clone, usando-se o valor genético real, obtendo-se assim o ganho real. Nessa sequência, estimou-se o ganho com seleção pelo BLUP e BLUP-HET. Posteriormente, comparou-se o ganho com seleção e a ordem dos melhores clones reais com a classificação dos melhores clones e seus respectivos ganhos por procedimento. Neste caso, os clones não são, necessariamente, os mesmos. Para verificar a coincidência existente entre os valores reais e os estimados, realizou-se uma contagem de genótipos comuns ocorrendo entre os 10 selecionados, para a estimativa real $\mathrm{x}$ BLUP, real $\mathrm{x}$ BLUP-HET e BLUP $\mathrm{x}$ BLUP-HET.

\section{Resultados e discussão}

Os resultados médios por variável (população simulada) das herdabilidades, acurácias estimadas pelos procedimentos BLUP e BLUP-HET e acurácia esperada são apresentados na Tabela 2.

As herdabilidades das populações simuladas variaram entre $3 \%$ e $24 \%$, com herdabilidade média de $11 \%$. A variação existente nas herdabilidades se deve à aleatoriedade na geração dos dados e à heterogeneidade das variâncias residuais, levando-se em consideração a variância genética de 0,10 . Rossetti (2002) ressalta que o maior número de repetições aumenta a precisão do experimento, propiciando maior confiabilidade. As amplitudes existentes entre as herdabilidades estimadas foi maior nos casos com duas repetições, reduzindo gradativamente, com o aumento do número de blocos. Esta amplitude demonstra a menor eficiência da estimação da herdabilidade em experimentos com dois ou cinco blocos. A partir de dez blocos, a amplitude foi inferior a $10 \%$, reduzindo com o aumento do número de repetições, mostrando-se mais eficiente.

Ocorreu um acréscimo no valor das acurácias seletivas em função do aumento do número de repetições, conforme esperado, com valores médios na faixa de $45 \%$ no caso de duas repetições para cerca de $76 \%$ com dez repetições. Nestas condições, a utilização de um número de blocos reduzidos influencia direta e negativamente nas estimativas, ocasionando maiores erros e, consequentemente, menores acurácias. Assim, seriam necessários pelo menos dez repetições para a obtenção de uma seleção mais confiável. Quando se analisa os dados por número de repetições, no caso de dois blocos, o procedimento BLUP foi superior ao BLUPHET em $80 \%$ dos casos. O BLUP-HET foi superior em $10 \%$ e, no caso da variável 4 , praticamente não houve diferença entre as acurácias. Os procedimentos BLUP e BLUP-HET apresentaram acurácias médias de $48 \%$ e $42 \%$, respectivamente. Apesar da pequena superioridade do BLUP, a acurácia é muito baixa para ambos os métodos, não sendo adequada para a seleção em casos com dois blocos. A inferioridade do BLUP-HET revela que apenas duas observações são inadequadas para estimar a variância de cada genótipo. 
Tabela 2. Valores de herdabilidade $\left(\mathrm{h}^{2}\right)$, média geral do experimento, correlação (BLUP x BLUP-HET), acurácias esperadas e acurácias estimadas pelos procedimentos BLUP e BLUP-HET, para as dez variáveis simuladas com 2, 5, 10 e 20 blocos.

\begin{tabular}{|c|c|c|c|c|c|c|c|c|c|c|c|c|}
\hline 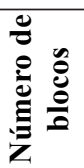 & Parâmetro & V1 & $\mathbf{V} 2$ & V3 & V4 & V5 & V6 & V7 & V8 & V9 & V10 & Média \\
\hline \multirow{6}{*}{ 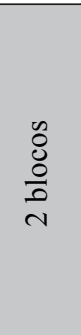 } & $\mathrm{h}^{2}$ (geral) & 0,23 & 0,10 & 0,17 & 0,24 & 0,14 & 0,17 & 0,03 & 0,22 & 0,12 & 0,08 & 0,15 \\
\hline & Média geral & 10,03 & 9,98 & 9,99 & 10,07 & 10,04 & 9,84 & 9,94 & 9,90 & 9,98 & 10,12 & 9,99 \\
\hline & Acurácia BLUP & 0,54 & 0,43 & 0,40 & 0,45 & 0,51 & 0,47 & 0,46 & 0,46 & 0,55 & 0,54 & 0,48 \\
\hline & Acurácia BLUP-HET & 0,41 & 0,36 & 0,43 & 0,46 & 0,45 & 0,37 & 0,33 & 0,42 & 0,49 & 0,45 & 0,42 \\
\hline & Correlação & 0,89 & 0,80 & 0,89 & 0,93 & 0,89 & 0,85 & 0,77 & 0,88 & 0,84 & 0,80 & 0,89 \\
\hline & Acurácia esperada & 0,61 & 0,42 & 0,54 & 0,62 & 0,50 & 0,54 & 0,23 & 0,60 & 0,46 & 0,39 & 0,49 \\
\hline \multirow{6}{*}{$\begin{array}{l}0 \\
0 \\
0 \\
0 \\
0 \\
n\end{array}$} & $\mathrm{~h}^{2}$ (geral) & 0,17 & 0,08 & 0,12 & 0,16 & 0,06 & 0,15 & 0,09 & 0,16 & 0,10 & 0,09 & 0,12 \\
\hline & Média geral & 9,97 & 9,99 & 9,95 & 9,95 & 10,00 & 10,05 & 9,97 & 10,03 & 10,03 & 10,09 & 10,00 \\
\hline & Acurácia BLUP & 0,67 & 0,64 & 0,58 & 0,68 & 0,68 & 0,66 & 0,65 & 0,67 & 0,67 & 0,52 & 0,64 \\
\hline & Acurácia BLUP-HET & 0,63 & 0,61 & 0,54 & 0,69 & 0,62 & 0,65 & 0,58 & 0,66 & 0,63 & 0,50 & 0,61 \\
\hline & Correlação & 0,97 & 0,93 & 0,95 & 0,96 & 0,90 & 0,96 & 0,93 & 0,97 & 0,93 & 0,93 & 0,97 \\
\hline & Acurácia esperada & 0,71 & 0,56 & 0,64 & 0,70 & 0,48 & 0,69 & 0,57 & 0,70 & 0,60 & 0,57 & 0,62 \\
\hline \multirow{6}{*}{$\begin{array}{l}0 \\
0 \\
0 \\
0 \\
0 \\
0\end{array}$} & $\mathrm{~h}^{2}$ (geral) & 0,12 & 0,07 & 0,10 & 0,10 & 0,13 & 0,12 & 0,05 & 0,09 & 0,12 & 0,08 & 0,10 \\
\hline & Média geral & 9,97 & 10,07 & 9,93 & 10,03 & 9,99 & 9,95 & 9,99 & 9,98 & 10,05 & 9,98 & 9,99 \\
\hline & Acurácia BLUP & 0,85 & 0,78 & 0,78 & 0,77 & 0,76 & 0,79 & 0,75 & 0,71 & 0,79 & 0,71 & 0,77 \\
\hline & Acurácia BLUP-HET & 0,84 & 0,77 & 0,78 & 0,79 & 0,75 & 0,76 & 0,73 & 0,71 & 0,78 & 0,70 & 0,76 \\
\hline & Correlação & 0,99 & 0,98 & 0,99 & 0,99 & 0,98 & 0,99 & 0,96 & 0,98 & 0,99 & 0,97 & 0,99 \\
\hline & Acurácia esperada & 0,76 & 0,67 & 0,73 & 0,72 & 0,77 & 0,76 & 0,60 & 0,70 & 0,76 & 0,68 & 0,72 \\
\hline \multirow{6}{*}{$\begin{array}{l}0 \\
0 \\
0 \\
0 \\
0 \\
0\end{array}$} & $\mathrm{~h}^{2}$ (geral) & 0,08 & 0,11 & 0,11 & 0,09 & 0,06 & 0,11 & 0,12 & 0,08 & 0,05 & 0,09 & 0,09 \\
\hline & Média geral & 9,99 & 9,99 & 10,03 & 9,94 & 10,04 & 9,93 & 10,01 & 9,96 & 9,98 & 9,99 & 9,98 \\
\hline & Acurácia BLUP & 0,85 & 0,87 & 0,83 & 0,83 & 0,75 & 0,83 & 0,89 & 0,85 & 0,79 & 0,85 & 0,84 \\
\hline & Acurácia BLUP-HET & 0,84 & 0,86 & 0,83 & 0,82 & 0,75 & 0,82 & 0,88 & 0,85 & 0,78 & 0,85 & 0,83 \\
\hline & Correlação & 0,99 & 1,00 & 1,00 & 1,00 & 0,99 & 1,00 & 0,99 & 0,99 & 0,99 & 0,99 & 0,99 \\
\hline & Acurácia esperada & 0,80 & 0,85 & 0,85 & 0,82 & 0,76 & 0,85 & 0,85 & 0,80 & 0,73 & 0,81 & 0,81 \\
\hline
\end{tabular}

Para a análise com cinco repetições, o procedimento BLUP foi superior ao BLUP-HET em $60 \%$ dos casos e, nos demais, os procedimentos foram semelhantes. A acurácia do BLUP foi de $64 \%$ e a do BLUP-HET foi de $61 \%$. Esta diferença a favor do BLUP provavelmente ocorreu devido à baixa precisão da estimativa das variâncias residuais dentro de clones estimada pelo procedimento BLUP-HET. Essa estimação com pequeno tamanho amostral via procedimento de quadrados mínimos parece inadequada, neutralizando a superioridade conceitual do BLUP-HET, que estima os componentes de variância por genótipo. Nestas condições, novos métodos de estimação da variância residual dentro de clones são necessários para melhor considerar essa heterogeneidade, principalmente para casos com número de repetições variando entre 5 e 10 . Procedimento como o de Foulley \& Quaas (1995) devem ser experimentados nesses casos.

Com dez repetições, apenas $10 \%$ das análises apresentaram diferenças, sendo o BLUP melhor neste caso. As acurácias médias do BLUP e BLUP-HET foram de $77 \%$ e $76 \%$, respectivamente, ou seja, praticamente iguais.

Nas análises com 20 repetições, os procedimentos foram semelhantes, com acurácias médias em torno de $84 \%$. Considerando a acurácia calculada por procedimento, o BLUP foi melhor no caso com dois e cinco blocos, sendo, a partir de dez blocos, praticamente 
iguais. Apesar da superioridade do BLUP com menor número de blocos, ocorreu uma menor precisão das estimativas, não sendo adequado o uso de um número reduzido de repetições com uma planta por parcela.

Nestes casos, para um caráter com herdabilidade na faixa de $10 \%$, a utilização de dez ou mais repetições não representa problema prático com heterogeneidade de variâncias residuais, podendo ser utilizado para análise qualquer um dos dois métodos.

Apesar de o BLUP ter sido superior ao BLUPHET para baixo número de repetições, a acurácia do procedimento BLUP-HET mostrou-se mais próximo à acurácia esperada para a maioria dos casos. No caso de duas repetições, $50 \%$ das acurácias estimadas foram mais próximas da acurácia esperada para cada procedimento, com melhor média do BLUP. Com cinco repetições, a acurácia estimada pelo BLUP foi mais próxima daacurácia esperada em $40 \%$ dos casos, contra $50 \%$ do
BLUP-HET e na média das dez variáveis. Com dez e vinte blocos, o BLUP apresentou acurácia estimada mais próxima a acurácia esperada apenas em $20 \%$ dos casos, tendo o BLUP-HET apresentado melhores resultados em $60 \%$ dos casos e na média das 10 variáveis. Levandose em consideração a maior proximidade da acurácia calculada com a acurácia esperada, o uso de duas e cinco repetições foram praticamente iguais, sendo que, para dez ou mais repetições, o procedimento BLUPHET mostrou-se mais próximo ao valor esperado. Isto indicaria o BLUP-HET como o método preferido para o caso de dez repetições. No entanto, as correlações entre os procedimentos BLUP e BLUP-HET foram de $99 \%$, indicando que ambos conduzem aos mesmos resultados.

A média e o desvio padrão das variâncias residuais estimadas pelo procedimento BLUP-HET por número de blocos e das herdabilidades são apresentados na Tabela 3.

Tabela 3. Média e desvio padrão das variâncias residuais dentro de clones e das herdabilidades médias dos clones estimados pelo procedimento BLUP-HET para 2, 5, 10 e 20 blocos.

\begin{tabular}{|c|c|c|c|c|c|c|c|c|c|c|c|}
\hline $\begin{array}{l}\text { Número de } \\
\text { blocos }\end{array}$ & V1 & $\mathbf{V} 2$ & $\mathbf{V 3}$ & V4 & V5 & V6 & V7 & V8 & V9 & V10 & Média \\
\hline \multicolumn{12}{|c|}{ Média das Variâncias Residuais } \\
\hline 2 & 0,61 & 0,72 & 0,64 & 0,62 & 0,66 & 0,62 & 0,80 & 0,58 & 0,61 & 0,66 & 0,65 \\
\hline 5 & 0,67 & 0,74 & 0,68 & 0,73 & 0,82 & 0,57 & 0,74 & 0,70 & 0,67 & 0,69 & 0,70 \\
\hline 10 & 0,68 & 0,68 & 0,77 & 0,70 & 0,66 & 0,70 & 0,72 & 0,66 & 0,70 & 0,70 & 0,70 \\
\hline 20 & 0,72 & 0,66 & 0,68 & 0,65 & 0,67 & 0,67 & 0,72 & 0,76 & 0,73 & 0,73 & 0,70 \\
\hline \multicolumn{12}{|c|}{ Desvio Padrão das Variâncias Residuais } \\
\hline 2 & 0,89 & 1,16 & 1,01 & 0,91 & 1,02 & 0,82 & 1,11 & 0,84 & 0,80 & 0,91 & 0,95 \\
\hline 5 & 0,51 & 0,49 & 0,51 & 0,52 & 0,50 & 0,39 & 0,52 & 0,41 & 0,57 & 0,50 & 0,49 \\
\hline 10 & 0,32 & 0,33 & 0,40 & 0,38 & 0,31 & 0,32 & 0,35 & 0,33 & 0,36 & 0,41 & 0,35 \\
\hline 20 & 0,25 & 0,24 & 0,27 & 0,22 & 0,22 & 0,23 & 0,26 & 0,38 & 0,24 & 0,28 & 0,26 \\
\hline \multicolumn{12}{|c|}{ Média das Herdabilidades } \\
\hline 2 & 0,49 & 0,41 & 0,44 & 0,48 & 0,40 & 0,42 & 0,26 & 0,48 & 0,38 & 0,32 & 0,41 \\
\hline 5 & 0,26 & 0,16 & 0,21 & 0,25 & 0,12 & 0,23 & 0,17 & 0,24 & 0,19 & 0,16 & 0,20 \\
\hline 10 & 0,17 & 0,11 & 0,14 & 0,14 & 0,17 & 0,17 & 0,08 & 0,12 & 0,17 & 0,13 & 0,14 \\
\hline 20 & 0,11 & 0,15 & 0,15 & 0,12 & 0,09 & 0,14 & 0,15 & 0,11 & 0,07 & 0,12 & 0,12 \\
\hline \multicolumn{12}{|c|}{ Desvio Padrão das Herdabilidades } \\
\hline 2 & 0,33 & 0,34 & 0,31 & 0,31 & 0,32 & 0,32 & 0,31 & 0,34 & 0,31 & 0,31 & 0,32 \\
\hline 5 & 0,16 & 0,13 & 0,14 & 0,15 & 0,11 & 0,14 & 0,14 & 0,15 & 0,12 & 0,11 & 0,14 \\
\hline 10 & 0,07 & 0,04 & 0,06 & 0,06 & 0,07 & 0,07 & 0,05 & 0,05 & 0,08 & 0,07 & 0,06 \\
\hline 20 & 0,04 & 0,05 & 0,05 & 0,04 & 0,04 & 0,05 & 0,04 & 0,03 & 0,02 & 0,04 & 0,04 \\
\hline
\end{tabular}


As variâncias residuais apresentaram médias semelhantes nas dez variáveis. Porém, quando se observa o desvio padrão das variâncias residuais, constata-se o aumento dos desvios com a redução do número de repetições, revelando que as variâncias residuais dentro de clones são estimadas de forma imprecisa com pequeno número de repetições. Com dez ou mais repetições esses desvios padrões são de baixa magnitude e as variâncias residuais dentro de clones são estimadas de forma mais precisa. Segundo Marion et al. (2001), a heterogeneidade nos componentes de variância reflete diretamente nas estimativas dos coeficientes de herdabilidade.

Os desvios padrões e as herdabilidades médias apresentaram, de maneira geral, tendência de redução dos valores com o aumento do número de blocos. Esta redução se deve à melhor estimação da herdabilidade média com o maior número de blocos, demonstrando que, para um pequeno número de repetições, as estimativas não são precisas.

As altas magnitudes dos desvios padrões tanto da variância residual quanto da herdabilidade de cada clone, especialmente para o caso de pequeno número de repetições, revela que os dados foram gerados sob forte heterogeneidade de variância residual, conforme premissa desejada. Em geral, os procedimentos padrões assumem variâncias residuais homogêneas para todos os tratamentos (Bernardo, 2002), o que ocasiona estimativas menos precisas.

A Tabela 4 apresenta os valores médios para a herdabilidade e variância assumida no momento da geração dos dados e para as herdabilidades e variâncias estimadas, de acordo com os números dos clones e número de repetições. As herdabilidades e as variâncias residuais tendem a se aproximar dos valores assumidos na geração, quando se aumenta o número de repetições. Nas análises com dois e cinco blocos, estes parâmetros foram estimados com baixa eficiência, enquanto que, nas análises com dez e vinte blocos, os resultados foram mais próximos aos esperados. Com base nestes resultados, observa-se que a estimação, tanto das herdabilidades quanto das variâncias residuais, foram ineficientes para um pequeno número de blocos, concordando com os resultados obtidos por Marion et al. (2001) e Rossetti (2002), em que os melhores resultados foram obtidos com dez ou mais blocos.

Tabela 4. Herdabilidade média $\left(h^{2}\right)$ e variância residual média $\left(\sigma^{2}\right)$ estimada por número de repetição e por clones obtidos através da simulação e herdabilidade esperada $\left(\mathrm{h}_{\text {esp }}^{2}\right)$ e variância residual esperada $\left(\sigma_{\text {esp }}^{2}\right)$, de acordo com a geração dos dados.

\begin{tabular}{cccccccccccc}
\hline \multirow{2}{*}{ Clones } & \multicolumn{2}{c}{ 2 Blocos } & \multicolumn{2}{c}{ 5 Blocos } & \multicolumn{2}{c}{ 10 Blocos } & \multicolumn{2}{c}{ 20 Blocos } & \multicolumn{2}{c}{ Valores assumidos } \\
\cline { 2 - 11 } & $\mathrm{h}^{2}$ & $\sigma^{2}$ & $\mathrm{~h}^{2}$ & $\sigma^{2}$ & $\mathrm{~h}^{2}$ & $\sigma^{2}$ & $\mathrm{~h}^{2}$ & $\sigma^{2}$ & $\mathrm{~h}^{2}{ }_{\text {esp }}$ & $\sigma^{2}{ }_{\text {esp }}$ \\
\hline 1 a 5 & 0,33 & 0,89 & 0,15 & 1,01 & 0,11 & 0,93 & 0,09 & 0,92 & 0,10 & 0,95 \\
6 a 25 & 0,37 & 0,77 & 0,17 & 0,81 & 0,12 & 0,81 & 0,11 & 0,77 & 0,11 & 0,80 \\
26 a 75 & 0,42 & 0,62 & 0,20 & 0,67 & 0,14 & 0,70 & 0,12 & 0,71 & 0,13 & 0,70 \\
76 a 95 & 0,43 & 0,56 & 0,22 & 0,62 & 0,17 & 0,56 & 0,14 & 0,60 & 0,14 & 0,60 \\
96 a 100 & 0,38 & 0,58 & 0,23 & 0,55 & 0,17 & 0,51 & 0,17 & 0,47 & 0,17 & 0,50 \\
\hline
\end{tabular}

As estimativas do ganho genético considerando uma intensidade de seleção de $10 \%$ e classificando pela ordem dos maiores valores genéticos reais estão apresentadas na Tabela 5. Neste caso, a ordem estimada pelos procedimentos BLUP e BLUP-HET não foi considerada, ou seja, os valores obtidos por estes procedimentos levam em consideração a ordem real. Ramos et al. (1996) e Teixeira et al. (2002) ressaltam sobre o risco de redução no progresso genético esperado quando ocorre heterogeneidade das variâncias, pois se pode selecionar maior proporção de indivíduos de maior variabilidade fenotípica e não de maior valor genético. O procedimento BLUP-HET apresentou uma estimativa maior que a real apenas em duas das quarenta análises, para os respectivos clones. Nos demais casos, a estimativa dos clones, por esse procedimento foi subestimada. O procedimento BLUP apresentou valores subestimados em todas as análises em relação ao valor real e apenas para seis análises apresentou melhores estimativas que o BLUP-HET. Considerando estas condições, o procedimento BLUP-HET apresenta estimativas mais próximas do valor real do que o procedimento BLUP, desconsiderando a ordem de classificação das estimativas. 
Tabela 5. Ganho com seleção em porcentagem (gs\%), considerando os dez melhores clones classificados pelo valor real e seus valores estimados pelos procedimentos BLUP e BLUP-HET e pela classificação por procedimento.

\begin{tabular}{|c|c|c|c|c|c|c|}
\hline \multirow{2}{*}{$\begin{array}{l}\text { Número de } \\
\text { blocos }\end{array}$} & \multirow{2}{*}{ Variáveis } & \multicolumn{3}{|c|}{$\begin{array}{c}\text { Dez melhores clones pela classificação real e } \\
\text { estimada }\end{array}$} & \multicolumn{2}{|c|}{ Dez melhores clones por procedimente } \\
\hline & & REAL & BLUP & BLUP-HET & BLUP & BLUP-HET \\
\hline \multirow{11}{*}{2 blocos } & v1 & 6,565 & 3,138 & 4,106 & 4,548 & 8,099 \\
\hline & v2 & 5,541 & 1,396 & 2,822 & 2,349 & 8,396 \\
\hline & v3 & 4,919 & 2,215 & 5,386 & 3,789 & 8,636 \\
\hline & $\mathrm{v} 4$ & 5,184 & 3,536 & 5,737 & 4,946 & 8,495 \\
\hline & v5 & 5,780 & 2,223 & 4,324 & 3,735 & 7,031 \\
\hline & v6 & 5,631 & 1,815 & 2,495 & 3,571 & 6,398 \\
\hline & v7 & 5,105 & 0,303 & 0,884 & 0,781 & 6,305 \\
\hline & v8 & 5,630 & 2,270 & 3,802 & 4,230 & 8,793 \\
\hline & v9 & 5,489 & 1,437 & 2,987 & 2,817 & 7,123 \\
\hline & v10 & 5,709 & 0,931 & 1,598 & 1,879 & 6,326 \\
\hline & Média & 5,555 & 1,926 & 3,414 & 3,265 & 7,560 \\
\hline \multirow{11}{*}{5 blocos } & v1 & 5,905 & 4,219 & 4,674 & 5,136 & 6,091 \\
\hline & v2 & 5,562 & 2,377 & 2,328 & 3,207 & 3,172 \\
\hline & v3 & 4,424 & 2,012 & 1,824 & 3,946 & 5,147 \\
\hline & $\mathrm{v} 4$ & 6,071 & 4,057 & 4,578 & 5,581 & 6,567 \\
\hline & v5 & 5,625 & 1,274 & 1,899 & 2,321 & 4,101 \\
\hline & v6 & 4,750 & 2,007 & 2,494 & 4,027 & 4,873 \\
\hline & v7 & 5,427 & 2,518 & 2,956 & 3,252 & 3,580 \\
\hline & v8 & 5,681 & 2,524 & 2,951 & 4,612 & 5,158 \\
\hline & v9 & 5,406 & 2,387 & 3,223 & 3,278 & 4,608 \\
\hline & $\mathrm{v} 10$ & 5,964 & 1,689 & 1,924 & 2,670 & 4,120 \\
\hline & Média & 5,481 & 2,506 & 2,885 & 3,803 & 4,742 \\
\hline \multirow{11}{*}{10 blocos } & $\mathrm{v} 1$ & 6,537 & 3,868 & 3,713 & 4,650 & 4,817 \\
\hline & v2 & 5,928 & 2,267 & 2,325 & 3,041 & 3,311 \\
\hline & v3 & 5,292 & 3,216 & 3,315 & 4,195 & 4,675 \\
\hline & $\mathrm{v} 4$ & 5,704 & 3,434 & 3,674 & 3,789 & 3,947 \\
\hline & v5 & 4,773 & 2,725 & 3,094 & 3,967 & 4,419 \\
\hline & v6 & 5,807 & 3,228 & 2,860 & 4,760 & 4,857 \\
\hline & v7 & 4,530 & 2,011 & 2,119 & 2,630 & 2,777 \\
\hline & v8 & 5,415 & 2,580 & 2,798 & 3,483 & 3,661 \\
\hline & v9 & 6,033 & 3,523 & 3,729 & 4,377 & 4,928 \\
\hline & v10 & 5,561 & 3,539 & 4,018 & 4,043 & 4,434 \\
\hline & Média & 5,558 & 3,039 & 3,164 & 3,894 & 4,183 \\
\hline \multirow{11}{*}{20 blocos } & $\mathrm{v} 1$ & 5,331 & 3,176 & 3,369 & 4,174 & 4,585 \\
\hline & v2 & 5,288 & 4,617 & 4,859 & 5,085 & 5,438 \\
\hline & v3 & 5,535 & 3,646 & 3,674 & 4,788 & 4,930 \\
\hline & $\mathrm{v} 4$ & 5,345 & 4,280 & 4,336 & 4,660 & 4,884 \\
\hline & v5 & 4,550 & 1,989 & 2,053 & 2,972 & 3,016 \\
\hline & v6 & 5,090 & 3,987 & 4,029 & 4,705 & 4,811 \\
\hline & v7 & 5,633 & 4,571 & 4,443 & 4,614 & 4,554 \\
\hline & v8 & 4,080 & 2,714 & 2,765 & 3,627 & 3,833 \\
\hline & v9 & 4,359 & 2,270 & 2,429 & 2,885 & 3,217 \\
\hline & $\mathrm{v} 10$ & 5,281 & 3,929 & 3,860 & 4,343 & 4,274 \\
\hline & Média & 5,049 & 3,518 & 3,582 & 4,185 & 4,354 \\
\hline
\end{tabular}


As estimativas do ganho genético com seleção obtidas pelos procedimentos BLUP e BLUP-HET mostraram que os clones selecionados não são, necessariamente, os mesmos. Todas as análises pelo procedimento BLUP foram inferiores aos valores reais, ou seja, subestimando o ganho genético. O procedimento BLUP-HET superestimou as estimativas do ganho com seleção em 15 análises efetuadas, sendo em todas as análises com dois blocos, em quatro análises com cinco blocos e apenas em uma análise com 20 blocos. Para as demais análises, a estimativa do procedimento BLUP-HET foi inferior ao estimado pelo real. Esta superestimativa ocorreu principalmente nos casos com menor número de blocos, pois se tem uma menor precisão para estimar a heterogeneidade de variâncias e, consequentemente, uma menor precisão na estimativa da variância residual e herdabilidade individual. Comparando os dois procedimentos, o BLUP apresentou maiores estimativas que o BLUP-HET em apenas trêr casos, mostrando-se mais conservador. Apesar das diferenças existentes, o BLUP-HET apresentou resultados do ganho com seleção mais próximo ao valor real em 32 análises, sendo o procedimento mais adequado para análises com heterogeneidade de variâncias, concordando com as conclusões de Campêlo et al. (2001) em melhoramento animal, que, desconsiderar a heterogeneidade de variância, quando esta existe, pode afetar o ganho genético. Estes resultados corroboram com Bernardo (2002), que ressaltam a necessidade de estimativas confiáveis de parâmetros genéticos na predição dos valores genéticos.

Comparando o número de clones comuns entre os dez melhores (real), com os estimados via BLUP e BLUPHET e depois entre o número de clones comuns com os obtidos pelos procedimentos BLUP e BLUP-HET, considerando para todas as comparações o número de blocos por variável analisada (Tabela 6), constatou-se novamente que o número de blocos interferiu diretamente na seleção. $\mathrm{O}$ aumento do número de blocos ocasiona uma melhora na estimativa, aumentando o número de clones comuns, tanto entre o BLUP e o BLUP-HET quanto entre estes procedimentos e os valores reais. Observa-se que, para 2, 5, 10 e 20 blocos, o BLUP apresentou semelhança com os valores reais, em termos médios, de 3,6, 4,3, 5,6 e 5,9 clones comuns, respectivamente, enquanto que o BLUP-HET apresentou valores de 3,1, 3,9, 5,6 e 5,5 clones comuns, respectivamente.
Entre os procedimentos, ocorreu um aumento de coincidências com o acréscimo do número de blocos, sendo de 5,6, 6,9, 8,5 e 8,7 clones comuns para 2, 5, 10 e 20 blocos, respectivamente. Para Vinson (1987), os indivíduos podem ser selecionados de forma indevida se as variâncias forem assumidas como homogêneas. Neste caso, delineamentos com maior número de repetições tendem a apresentar resultados mais próximos entre os dois procedimentos. Apesar da pequena diferença no número de clones selecionados pelos dois procedimentos com a ordem dos valores genéticos reais, o BLUP apresentou uma pequena superioridade para baixo número de repetições.

Tabela 6. Número de clones comuns entre os dez melhores clones (real) e estimados (BLUP e BLUP-HET) e número de clones comuns entre os procedimentos BLUP e BLUP-HET, por número de blocos e por variável analisada.

\begin{tabular}{|c|c|c|c|c|c|c|}
\hline \multirow{2}{*}{ 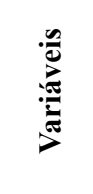 } & $\begin{array}{c}\text { Real } \\
\mathbf{X} \\
\text { BLUP }\end{array}$ & $\begin{array}{c}\text { Real } \\
X \\
\text { HET }\end{array}$ & $\begin{array}{c}\text { BLUP } \\
\mathbf{X} \\
\text { HET }\end{array}$ & $\begin{array}{c}\text { Real } \\
\mathbf{X} \\
\text { BLUP }\end{array}$ & $\underset{\text { HET }}{\text { Real }}$ & $\begin{array}{c}\text { BLUP } \\
\mathbf{X} \\
\text { HET }\end{array}$ \\
\hline & \multicolumn{3}{|c|}{2 Blocos } & \multicolumn{3}{|c|}{5 Blocos } \\
\hline v1 & 4 & 3 & 5 & 5 & 6 & 7 \\
\hline v2 & 3 & 2 & 6 & 6 & 4 & 6 \\
\hline v3 & 4 & 5 & 6 & 3 & 2 & 6 \\
\hline v4 & 4 & 4 & 5 & 6 & 4 & 6 \\
\hline v5 & 6 & 4 & 7 & 3 & 3 & 8 \\
\hline v6 & 5 & 4 & 6 & 3 & 4 & 7 \\
\hline v7 & 1 & 1 & 6 & 6 & 5 & 7 \\
\hline v8 & 4 & 3 & 4 & 3 & 4 & 9 \\
\hline v9 & 2 & 3 & 6 & 5 & 5 & 7 \\
\hline v10 & 3 & 2 & 5 & 3 & 2 & 6 \\
\hline \multirow[t]{2}{*}{ média } & 3,6 & 3,1 & 5,6 & 4,3 & 3,9 & 6,9 \\
\hline & \multicolumn{3}{|c|}{10 Blocos } & \multicolumn{3}{|c|}{20 Blocos } \\
\hline v1 & 7 & 6 & 8 & 6 & 6 & 9 \\
\hline v2 & 5 & 6 & 9 & 7 & 8 & 9 \\
\hline v3 & 6 & 4 & 8 & 5 & 5 & 9 \\
\hline v4 & 7 & 6 & 8 & 7 & 5 & 8 \\
\hline v5 & 5 & 5 & 10 & 3 & 3 & 9 \\
\hline v6 & 4 & 4 & 8 & 6 & 6 & 8 \\
\hline v7 & 5 & 6 & 7 & 9 & 8 & 9 \\
\hline v8 & 6 & 7 & 9 & 5 & 4 & 9 \\
\hline v9 & 5 & 5 & 10 & 5 & 4 & 8 \\
\hline $\mathrm{v} 10$ & 6 & 7 & 8 & 6 & 6 & 9 \\
\hline média & 5,6 & 5,6 & 8,5 & 5,9 & 5,5 & 8,7 \\
\hline
\end{tabular}




\section{Conclusões}

As estimativas realizadas com duas ou cinco repetições mostram-se de baixa precisão. Para o caso de herdabilidades em torno de $10 \%$, recomenda-se o uso de pelo menos dez repetições, o que resulta em desvios padrões de baixa magnitude e variâncias residuais estimadas com maior precisão, maximizando as acurácias.

Apesar do BLUP ter sido superior ao procedimento BLUP-HET no caso de pequeno número de repetições (2 a 5), na maioria dos casos, a acurácia média do BLUP-HET mostra-se mais próxima ao valor esperado, sendo que o BLUP-HET apresenta ganhos com seleção mais próximos do real.

Com herdabilidades em torno de $10 \%$ e a utilização de dez ou mais repetições, a heterogeneidade de variâncias dentro de genótipos não representa problema prático para o melhorista. Nestes casos, qualquer um dos procedimentos pode ser utilizado.

\section{Referências}

BERNARDO, R. Breeding for quantitative traits in plants. Woodbury: Stemma Press, 2002. 369 p.

CAMPÊLO, J. E. G.; LOPES, P. S.; TORRES, R. A.; EUCLYDES, R. F.; SILVA, L. O. C.; ARAÚJO, C. V.; PEREIRA, C. S. Influência da heterogeneidade de variância no ordenamento de reprodutores da raça tabapuã. Revista Científica de Produção Animal, v. 3, n. 1, p. 97-106, 2001.

CRUZ, C. D.; REGAZZI, A. J.; CARNEIRO, P. C. S. Modelos biométricos aplicados ao melhoramento genético. 3. ed. Viçosa, MG: UFV, 2004. 480 p. v. 1.

FELIPE, C. R. de P.; DUARTE, J. B. Comparison of estimation and prediction methods of genotypic means in maize variety trials. Crop Breeding and Applied Biotechnology, Londrina, v. 10, n. 2, p. 147-153, 2010.

FOULLEY, J. L.; QUAAS, R. L. Heterogeneous variances in gaussian linear mixed models. Genetics Selection Evolution, v. 27, p. 211-228, 1995

MARCELINO, S. D. R.; IEMMA, A. F. Métodos de estimação de componentes de variância em modelos mistos desbalanceados. Scientia Agrícola, v. 57, n. 4, p. 643-652, 2000.
MARION, A. E.; RORATO, P. R. N.; FERREIRA, G. B.; EVERLING, D. M. F.; FERNANDES, H. D. Estudo da heterogeneidade das variâncias para as características produtivas de rebanhos da raça holandesa no Rio Grande do Sul. Revista Brasileira de Zootecnia, v. 30, n. 6 (suplemento), p. 1995-2001, 2001.

RAMOS, A. A.; TÁPIA VALENCIA, E. F.; WECHSLER, F. S.; GONÇALVES, H. C. Heterogeneidade de variância das características produtivas de bovinos da raça holandesa nos trópicos: I - estratificação por nível de produção de rebanho. In: REUNIÃO ANUAL DA SOCIEDADE BRASILEIRA DE ZOOTECNIA, 33., 1996, Fortaleza, CE. Anais... Fortaleza, CE: SBZ, 1996. p. 71-73.

RESENDE, M. D. V. de. Selegen-Reml/Blup: sistema estatístico e seleção genética computadorizada via modelos lineares mistos. Colombo: Embrapa Florestas, 2007. 361 p.

RESENDE, M. D. V. Genética biométrica e estatística no melhoramento de plantas perenes. Brasília, DF: Embrapa Informação Tecnológica; Colombo: Embrapa Florestas, 2002. 975 p.

RESENDE, M. D. V. Delineamento de experimentos de seleção para a maximização da acurácia seletiva e do progresso genético. Revista Árvore, v. 19, n. 4, p. 479-500, 1995.

RESENDE, M. D. V.; DUARTE, J. B. Precisão e controle de qualidade em experimentos de avaliação de cultivares. Pesquisa Agropecuária Tropical, v. 37, n. 3, p. 182-194, 2007.

RÖNNEGARD, L.; FELLEKI, M.; FIKSE, F.; MULDER, H. A.; STRANDBERG, E. Genetic heterogeneity of residual variance: estimation of variance components using double hierarchical generalized linear models. Genetics Selection Evolution, v. 42, n. 8, p. 1-10, 2010. DOI 10.1186/1297-9686-42-8.

ROSSETTI, A. G. Influência da área da parcela e do número de repetições na precisão de experimentos com arbóreas. Pesquisa Agropecuária Brasileira, v. 37, n. 4, p. 433-438, 2002.

TEIXEIRA, N. M.; FREITAS, A. F.; FERREIRA, W. J.; DURÃES, M. C.; BARRA, R. B. Ajustamento para heterogeneidade de variância da produção de leite de vacas da raça holandesa no Estado de Minas Gerais. Revista Brasileira de Zootecnia, v. 31, n. 1 (suplemento 1), p. 369-375, 2002.

VINSON, W. E. Potential bias in genetic evaluations from differences in variation within herds. Journal Dairy Science, v. 70, p. 2450-2455, 1987. 